Working

Paper

Department

of Economics

$\mathrm{Ca}^{\prime}$ Foscari University of

Venice

Sergio Currarini

Networks Design in

Games with Spillovers 


\title{
Network Design in Games with Spillovers
}

\author{
Sergio Currarini \\ University of Venice
}

\begin{abstract}
How should an organization be designed in order to provide its members with minimal incentives to defect? And how does the optimal design depend on the type of strategic interaction between defectors and remaining organizational members? This paper addresses such issues in a game theoretic model of cooperation, in which an organization is formally represented by a connected network, and where gains from cooperation are given by a partition function. We show that critical structural features of the organization depend in a clear-cut way on the sign of spillovers. In particular, positive spillovers favor the adoption of dispersed and centralized forms, while negative spillovers favor cohesive and horizontal ones. Moreover, if the organizational form determines all the communication possibilities of members, a highly centralized organization - the star - emerges under positive spillovers, whereas two horizontal architectures - the circle and the complete - emerge under negative spillovers.
\end{abstract}

\section{Keywords}

Organizational design, networks, group stability, spillovers.

\section{JEL Codes}

C7, C71, D20.

$$
\begin{array}{r}
\text { Address for correspondence: } \\
\text { Sergio Currarini } \\
\text { Department of Economics } \\
\text { Ca' Foscari University of Venice } \\
\text { Cannaregio 873, Fondamenta S.Giobbe } \\
30121 \text { Venezia - Italy } \\
\text { Phone: }(++39) 0412349133 \\
\text { Fax: (++39) } 0412349176 \\
\text { e-mail: s.currarini@unive.it }
\end{array}
$$

This Working Paper (o QD se in inglese) is published under the auspices of the Department of Economics of the Ca' Foscari University of Venice. Opinions expressed herein are those of the authors and not those of the Department. The Working Paper series (o OD se in inglese) is designed to divulge preliminary or incomplete work, circulated to favour discussion and comments. Citation of this paper should consider its provisional character.

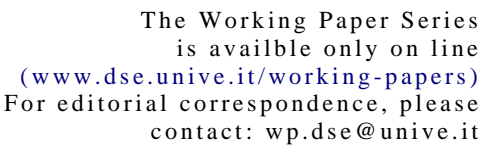

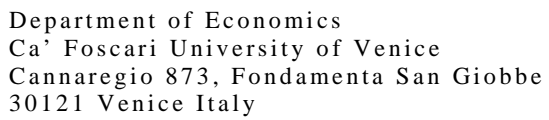

Department of Economics

Ca' Foscari University of Venice

Cannaregio 873, Fondamenta San Giobbe

30121 Venice Italy

Fax: ++390412349210 


\section{Introduction}

The existence of gains from cooperation, and the potential failure of economic agents to capture such gains, are a recurrent feature of many economic and social situations. The game theoretic analysis of cooperation has traditionally focused on the notion of "coalition", by this meaning a set of agents acting towards a common collective interest. In a seminal contribution, Myerson (1977) has pointed out how the notion of cooperation structure (or graph or network), can greatly enrich the analysis by fully describing the set of bilateral relations underlying the emergence of cooperation. In Myerson's framework, a network that allows all agents to communicate, either directly or indirectly through other agents, can be viewed as an "organization", specifying a set of cooperating members and an "organizational form" through which the coordination of individual actions is attained.

Depending on the problem at stake and on the time horizon one considers, cooperation networks can display varying degrees of flexibility. For instance, social organizations are shaped by interpersonal relations based on trust or kinship, which are by nature rigid or very costly to create and destroy. The same applies to networks originating from customs, tradition, ideological positions or previous commitment, or from the design of a central authority. By contrast, in certain forms of economic collaborations (such as cartels, R\&D collaborations or international agreements), agents are usually free to modify their links at any time and at low costs. In this paper we will be concerned with the first type of rigid and non modifiable networks.

The consideration of cooperation networks rather than coalitions raises a series of theoretical questions concerning the stability of cooperation. How does the organizational form affect the incentives and the ability of members to defect and to take independent actions? Are certain architectures intrinsically more stable than others? Are, for instance, strongly cohesive organizations always more stable than more dispersed ones? Is centralization always good for stability?

In order to get some insight into these complex issues, we will address the problem of stability from a specific angle, in line with the tradition of cooperative game theory. We will take as a primitive concept the gains from cooperation that a coalition of players can enjoy when embedded in a given "coalition structure", by this meaning a configuration of all coalitions active in the system. These gains will be assumed independent of the internal organization adopted by such coalitions, and to depend only on their external configuration. 
Although this is certainly a drastic simplification, overlooking all efficiency consideration that motivate a large part of organization theory ${ }^{1}$, it allows us to isolate the effect of the network on the stability of cooperation. Moreover, by allowing the gains from cooperation to depend on the whole coalition structure, we can include in our analysis problems in which the formation or disintegration of coalitions have welfare effects (or "spillovers") on potentially all players in the system, such as public goods economies, industrial oligopolies, trade blocks, etc... ${ }^{2}$. We will talk of positive spillovers (resp., negative spillovers) when the formation of a coalition (or the merger of several coalitions) benefits (resp., hurts) the other agents in the system (see Yi (1997)).

A first set of results we obtain are driven by the way in which such spillovers shape the incentives of organizational members to defect from different architectures. The main ingredient, and the novelty, of the present analysis is the assumption that the way in which members are connected within the organization can be used to predict the degree of cooperation that would follow any coalitional defection from the organization. Because of the presence of spillovers, such predictions are crucial to assess the profitability of such defections. Consider for instance a political alliance, in which parties $\mathrm{B}$ and $\mathrm{C}$ can cooperate on a common programme only thanks to the mediation of A (this may happen, for instance, because of the distance between B's and C's ideological positions). By quitting the alliance, $\mathrm{A}$ is likely to face $\mathrm{B}$ and $\mathrm{C}$ as singletons in the political competition. Party A would face a different perspective if $\mathrm{B}$ and $\mathrm{C}$ had closer ideological positions, in which case a defection of A may be followed by the formation of an alliance of B and C. Party A is likely to find the defection more profitable in the first case, due to the negative spillovers of political competition. An opposite and symmetric story would apply in a public goods economy, where spillovers from coalition formation are positive. Here, player A would enjoy the benefits of free riding on $\mathrm{B}$ and $\mathrm{C}$ only if these were linked in the network, otherwise A's defection would lead to a complete absence of cooperation.

Propositions 1 and 2 generalize the simple mechanics outlined above, providing some basic insights into the role played by the sign of the spillovers in determining which network is less vulnerable to defections. In particular, these results relate the cohesion of an organization

\footnotetext{
${ }^{1}$ See, among others, thr seminal work of Chandler (1966) and, more recently, of Arrow (1974), Radner (1993), Bolton and Dewatripont (1994), Holmstrom and Milgrom (1991, 1994).

${ }^{2}$ See Yi (2003) and Bloch (2003) for recent surveys of the vast literature on cooperative games with spillovers.
} 
to its stability properties, and shows that positive and negative spillovers lead to opposite conclusions. In particular, sparser organizational forms (in our simplified framework, trees) are more stable under positive spillovers, while more cohesive ones (in our framework, the complete network) are more stable under negative.

A second set of results accounts for a second major force relating the network architecture (that is, the organizational form) with the stability of cooperation. In addition to affecting the incentives to defect, the organizational form can indeed determine the communication possibilities of members, and, therefore, their ability to defect from the organization in a coordinated manner. In the above example, for instance, in the absence of a direct link parties B and C may find it impossible to communicate if not through A. Unable to make coordinated plans, $\mathrm{B}$ and $\mathrm{C}$ would not pose a threat to the alliance as a defecting coalition. This effect of the network on the "ability" of coalitions to defect has been shown by Demange (2004) to induce stability in all superadditive problems without spillovers. ${ }^{3}$ In this paper, it allows for sharp results for the specific case in which the gains from cooperation are equally shared among cooperating agents. In such egalitarian problems, a single minimally connected network - the star - is selected as the most stable organizational form in all problems with positive spillovers. When spillovers are negative, two architectures are selected, with equivalent stability properties: the complete network, in which all pairs are linked, and the circle, in which all members maintain two links.

The paper is organized as follows. Section 2 introduces the basic concepts and notation. Section 3 defines the notion of stable allocations and stable organizations, presents the main results and relates them to the design of stable organizations. Section 4 concludes the paper.

\section{The Model}

\subsection{Coalitions, Values and Allocations}

We will be considering a set $N$ of $n$ players, with generic element $i=1,2, \ldots n$. A coalition of players is any subset $S \subseteq N$; in particular, the set $N$ is referred to as the grandcoalition. A partition $\pi_{S}$ of $S \subseteq N$ is a collection $\left\{B_{1}, B_{2}, \ldots, B_{m}\right\}$ of subsets of $S$ such that $B_{k} \cap B_{l}=\emptyset$ for all $k \neq j$, and such that $\cup_{p=1}^{m} B_{p}=S$. A partition $\pi_{S}$ of $S$ is also called a coalition structure on $S$. For all $S \subseteq N$, the set of all partitions of $S$ is denoted by $\Pi(S)$. The pair $(S, \pi)$ such

\footnotetext{
${ }^{3}$ In non superadditive problems, some stable configuration of coalitions is also shown to exist.
} 
that $S \in \pi \in \Pi(N)$ is called an embedded coalition. For all $(S, \pi)$, we denote by $\pi \backslash S$ the partition of the set $N \backslash S$ obtained by considering the elements of $\pi$ except $S$.

A partition function $v$ associates with each embedded coalition $(S, \pi)$ a real number $v(S, \pi)$. Intuitively, the grandcoalition represents a situation where all players in $N$ cooperate in order to achieve the maximal aggregate welfare; a coalition structure $\pi$ on $N$ indicates that several distinct coalitions have formed, within which players cooperate, but across which some kind of noncooperative strategic interaction takes place. ${ }^{4}$ So, the value $v(S, \pi)$ indicates the maximal aggregate payoff achievable by the members of $S$ when interacting with the other elements of $\pi$.

The following assumptions impose restrictions on the type of strategic interaction taking place within different coalition structures; such assumptions are formulated as properties of the partition function $v$.

We start with the assumption of anonymity. A permutation $\sigma$ is a bijection from $N$ into $N$ (a relabelling of the set of players). The notation $\sigma(S)$ will indicate the new coalition obtained by relabelling the members of $S$ according to $\sigma$, and $\sigma(\pi)$ to indicate the new partition obtained by relabelling the players in the elements of $\pi$ according to $\sigma$.

Definition 1 The partition function $v$ is anonymous if for all permutations $\sigma$ of the set $N$, and for all embedded coalitions $(S, \pi)$, we have $v(S, \pi)=v(\sigma(S), \sigma(\pi))$.

We say that $v$ is cohesive if the grandcoalition generates more value than any of its partitions (there are no decreasing returns to cooperation).

Definition 2 The partition function $v$ is cohesive if $v(N,\{N\}) \geq \sum_{B \in \pi} v(B, \pi)$ for all $\pi \in$ $\Pi(N)$.

A stronger assumption, called full cohesiveness and not used in the present paper, requires that the same property applies to all embedded coalitions.

The next properties of $v$ classify strategic interaction among coalitions in terms of the change in the value $v(S, \pi)$ that occurs in response to changes in the configuration of the sub-partition $\pi \backslash S$. This classification, proposed in Yi (1997), makes use of the preliminary notion of "concentration" of a partition. ${ }^{5}$

\footnotetext{
${ }^{4}$ See Ichiischi (1981) for a concept of strategic equilibrium associated to a coalition structure.

${ }^{5}$ This definition is weaker than that used by Yi, but is sufficient for the results of the present paper.
} 
Definition 3 Let $S \subseteq N$. The partition $\pi \in \Pi(S)$ is a concentration of $\pi^{\prime} \in \Pi(S)$ if $\pi \neq \pi^{\prime}$ and if it is possible to originate $\pi$ by merging elements of $\pi^{\prime}$. Formally, $\pi$ is a concentration of $\pi^{\prime}$ if for all $B \in \pi$ either $B \in \pi^{\prime}$ or there exists a collection $\left\{B_{1}, . ., B_{p}\right\}$ of elements of $\pi^{\prime}$ such that $B=\bigcup_{k=1}^{p} B_{k}$.

Definition 4 The partition function $v$ exhibits positive spillovers if $v(S, \pi)>v\left(S, \pi^{\prime}\right)$ for all embedded coalitions $(S, \pi)$ and $\left(S, \pi^{\prime}\right)$ such that $\pi$ is a concentration of $\pi^{\prime}$.

Definition 5 The partition function $v$ exhibits negative spillovers if $v(S, \pi)<v\left(S, \pi^{\prime}\right)$ for all embedded coalitions $(S, \pi)$ and $\left(S, \pi^{\prime}\right)$ such that $\pi$ is a concentration of $\pi^{\prime}$.

Intuitively, positive spillovers arise when a coalition benefits from the merging of other coalitions (this happens, for instance, in public goods economies and in linear Cournot oligopoly). Negative spillovers arise when a coalition is hurt by the merging of other coalitions (this happens, for instance, in trade blocks problems and in R\&D alliances). We will denote by $\bar{V}$ the set of all partition functions, and by $V^{+}$and $V^{-}$the classes of partition function exhibiting positive and negative spillovers, respectively. It is clear that $V^{+} \cup V^{-} \subset \bar{V}$.

Finally, an allocation specifies the way in which the values assigned by $v$ to the coalitions in $\pi$ are distributed within these coalitions.

Definition 6 An allocation for the pair $(N, v)$ is a pair $(\mathbf{u}, \pi) \in R_{+}^{n} \times \Pi(N)$, where $\mathbf{u}$ is a vector of imputed payoffs for the set of players $N$ such that $\sum_{i \in B} \mathbf{u}_{i}=v(B, \pi)$ for all $B \in \pi$.

Note that if $v$ is cohesive, then whenever $(\mathbf{u}, \pi)$ is an allocation also $(\mathbf{u},\{N\})$ is an allocation. For this reason, whenever dealing with cohesive partition functions we will always refer to allocations of the form $(\mathbf{u},\{N\})$, that for simplicity we will simply denote by $\mathbf{u}$.

\subsection{Connected Networks as Organizations}

In this section we make use of the notion of network (or graph) to provide a detailed specification of the interpersonal bilateral relations that shape the internal organization of a coalition.

A network $g$ is given by a set of vertices $N(g)$ together with a set $L(g)$ of bilateral links between pairs of vertices (that is, unordered pairs of elements of $N(g)$ ). A link between the vertices $i$ and $j$ is denoted by $i j$. The network $g$ is complete if $i j \in L(g)$ for all $i$ and $j$ in 
$N(g), i \neq j$. The network $h$ is a subnetwork of $g$ (and we write $h \subset g$ ) if $N(h) \subseteq N(g)$ and $L(h) \subseteq L(g)$, with strict inclusion for at least one of the two sets.

Two agents $i$ and $j$ are connected in $g$ if there exists a set of vertices (a "connecting path") $\left\{i_{1}, i_{2}, \ldots, i_{k}\right\} \subseteq N(g)$ such that $i=i_{1}, j=i_{k}$, and $i_{p} i_{p+1} \in g$ for all $p=1, \ldots, k-1$. The set of vertices $S \subseteq N(g)$ is connected in $g$ if for all pairs of vertices in $S$ there exists a connecting path all contained in $S$. The set of connected coalitions in $g$ is denoted by $\mathcal{C}(g)$. The network $g$ is connected if $N(g) \in \mathcal{C}(g)$. The subnetwork $h$ of $g$ is a component of $g$ if $h$ is connected and if there exists no other connected subnetwork $h^{\prime}$ of $g$ such that $h \subset h^{\prime}$. The set of components of the network $g$ is denoted by $C(g){ }^{6}$

The set $C(g)=\left\{h_{1}, h_{2}, \ldots, h_{m}\right\}$ is made of $m$ connected networks with pairwise disjoint sets of vertices $N\left(h_{1}\right), N\left(h_{2}\right), \ldots, N\left(h_{m}\right)$. We can therefore associate with each network $g$ such that $N(g)=N$ the following unique partition $\pi(g) \in \Pi(N)$ :

$$
\pi(g)=\left\{N\left(h_{1}\right), N\left(h_{2}\right), \ldots, N\left(h_{m}\right)\right\}
$$

In this sense, a network $g$ provides a more detailed information than the associated partition $\pi(g)$, since it also specifies the organizational form adopted by each coalition through the architecture of the various components of $g .{ }^{7}$ This motivates the next definition.

Definition 7 An organization is a connected graph $g$. The set $N(g)$ is called the set of organizational members. The set of links $L(g)$ is called the organizational form of $g$.

In the analysis of the next section we will make use of some additional notation, allowing us to express the networks obtained by deleting vertices from a given network $g$ with set of vertices $N$. For $S \subset N$, we denote by $\left.g\right|_{S}$ the graph with vertex set $S$ obtained by deleting the set $N \backslash S$ of vertices from $g$; the set of links $L\left(\left.g\right|_{S}\right)$ is given by $\{i j \in g:\{i, j\} \subseteq S\}$.

If $g$ is connected but $\left.g\right|_{N \backslash\{i\}}$ is disconnected, then vertex $i$ is called a cut-vertex of $g$. If $g$ is connected but $\left.g\right|_{N \backslash S}$ is disconnected, then $S$ is called a vertex-cut of $g$.

Three connected architectures that will play a central role in our analysis are the star $g^{*}$, with one central vertex maintaining all links, the circle $g^{c}$, with all vertices organized on a circle, each maintaining two links, and the complete network $g^{N}$, containing all links.

\footnotetext{
${ }^{6}$ It is clear that $C(g) \subset \mathcal{C}(g)$.

${ }^{7}$ The association of coalitions with connected networks is implicit in Myerson's (1977) analysis; in fact, the "Myerson value" allocates to each connected component the worth of the coalition identified by its vertices.
} 

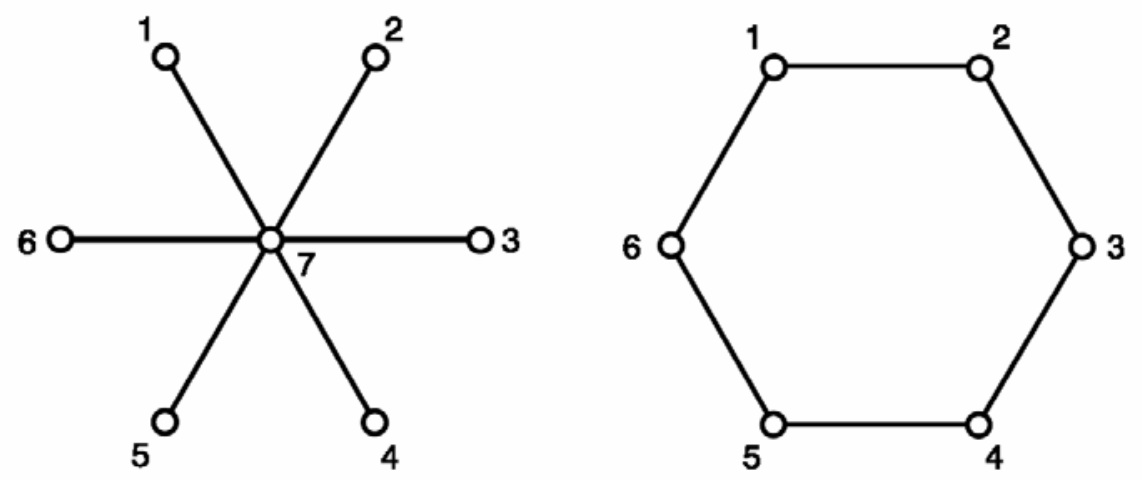

Star network with $n=7$ and circle network with $n=6$

\section{The Design of Stable Organizations}

In order to be stable, an organization must prevent all potential defections of its members. In order to threaten the stability of the organization, such defections must be profitable, by this meaning that they must induce a payoff allocation which is preferred by defectors to the payoff allocation adopted within the organization.

In this section we study how a hypothetical designer can shape the architecture of the cooperation network in order to prevent such defection by means of an appropriately chosen payoff allocation. We will only look at the stability of organizations with set of members $N$; although all results extend to the stability of smaller organizations (that is, on components of a network $g$ ), focusing on $N$ makes notation simpler. Section 3.1 looks at the effect of organizational design on the profitability of defections, characterizing the relation between the network and the outside options of the various coalitions. Section 3.2 looks at the relation between the network and the ability of various coalitions to defect.

\subsection{Incentives to defect from an organization}

Consider a coalition $S \subset N$ of members of the organization $g$, with $N(g)=N$, and assume that within $g$ the payoff allocation $\mathbf{u}$ has been chosen. In case of defection from $g$, the members of $S$ are free to adopt any coalition structure $\pi_{S}$ in order to obtain a higher payoff than at u. This "outside option" available to $S$ depends, through the effect of the spillovers, on the 
coalition structure that is induced on $N \backslash S$ by its defection from $g$. Central to our analysis is the assumption that the way in which players in $N \backslash S$ will form coalition depends on the way in which these players communicate in the organization $g$. More precisely, we assume that the coalition structure induced on $N \backslash S$ is uniquely determined by the components of the "residual" graph $\left.g\right|_{N \backslash S}$ with vertex set $N \backslash S$, obtained by deleting from $g$ all vertices in $S$. This approach can be justified as a form of myopic expectations of defecting coalitions, who expect the existing links to characterize cooperative relations as well in the future. Alternatively, it can be viewed as a formalization of the assumption that the network is rigid, and it takes time to establish new cooperative relations or to sever existing ones.

The partition of the set $N \backslash S$ associated with the defection of $S$ is therefore $\pi\left(\left.g\right|_{N \backslash S}\right)$. By defecting from the organization $g$ and adopting coalition structure $\pi_{S}$, coalition $S$ can therefore guarantee its members all payoff allocations which are feasible for the partition $\left\{\pi_{S}, \pi\left(\left.g\right|_{N \backslash S}\right)\right\}$ according to the partition function $v$. This leads us to the following definition.

Definition 8 Let $g$ be an organization with set of members $N$ and $v$ be a cohesive partition function. Coalition $S \subset N$ has the incentive to defect from $g$ under payoff allocation $\mathbf{u}$ and partition function $v$ if there exists $\pi_{S} \in \Pi(S)$ such that $v\left(B,\left\{\pi_{S}, \pi\left(\left.g\right|_{N \backslash S}\right)\right\}\right)>\sum_{i \in B} u_{i}$ for all $B \in \pi_{S}$.

\subsection{Ability to defect from an organization}

In this section we consider a second aspect of organizational design, related to the possibility that the adopted organizational form impose constraints on the ability of coalitions to communicate and coordinate without the help of the remaining members. Such constraints are likely to affect the ability of players to enact defections, which are typically the result of a series of coordinated activities, such as the assessment of the actions available outside the organization, the evaluation of the consequences of such actions, and their actual implementation. An analysis of such constraints in the context of cooperative games without spillovers is contained in Demange (2004), where the ability to defect from an organization $g$ is restricted to the class of coalitions which are internally connected in $g$. In the same spirit, we denote as restricted communication the situation where the set of coalition which are able to defect from $g$ is $\mathcal{C}(g)$.

The case of unrestricted communication is also important. All problems in which the players are able to communicate and make contingent plans before the adoption of a specific 
organizational form should belong to this latter class. The same applies to problems in which information is transmitted through the network independently of the will of the agents, so that any two players who are not directly linked are still able to jointly defect from the organization. In this case, the set of coalition which are able to defect from $g$ is the set of all subsets of $N$.

\subsection{Stable Allocations within an Organization}

Having described the incentives and the ability of coalitions to defect from an organization $g$, we are able to formalize the notion of a stable allocation.

Definition 9 The payoff allocation $\mathbf{u}$ is stable for the organization $g$ with set of members $N$ under the partition function $v$ if no coalition $S \subset N$ has both the ability and the incentive to defect from $g$.

We denote by $\Phi(g, v)$ the set of all stable allocations for $g$ under $v$.

If the partition function $v$ is fully cohesive, and the assumption of unrestricted communication is made, the set of stable allocations for $g$ can be expressed as the core of the game in characteristic function $(N, f(v, g))$, where:

$$
f(v, g)(S) \equiv v\left(S,\left\{S, \pi\left(\left.g\right|_{N \backslash S}\right)\right\}\right) .
$$

If $v$ is not fully cohesive, and the assumption of unrestricted communication is maintained, we can still obtain the set of stable allocations for $v$ as the core of a game in characteristic function without side payments, where the set of payoff allocations attainable by coalition $S$ is given by:

$$
F(v, g)(S) \equiv\left\{u_{S} \in R_{+}^{S}: \exists \pi_{S} \in \Pi(S): v\left(B,\left\{\pi_{S}, \pi\left(\left.g\right|_{N \backslash S}\right)\right\}\right) \geq \sum_{i \in B} u_{i} \forall B \in \pi_{S}\right\} .
$$

A stable organization is consequently defined by the existence of at least one stable allocation:

Definition 10 The organization $g$ with vertex set $N$ is stable under the partition function $v$ if $\Phi(g, v) \notin \varnothing$. 
Again, under the assumption of unrestricted communication a stable organization is one allowing for a nonempty core in the associated game in characteristic function.

The results that follow record a monotonicity property that applies to the two classes of partition functions with positive and negative spillovers. This property has straightforward implication for the design of stable organizations, that we discuss in the next section.

Consider the effect of deleting one or more links from the organization $g$, originating the organization $g^{\prime} \subset g$. It is immediate that for all $S \subset N$, the partition $\pi\left(\left.g\right|_{N \backslash S}\right)$ is either identical to or a concentration of the partition $\pi\left(\left.g^{\prime}\right|_{N \backslash S}\right)$ (see the proof of lemma 1 below). It follows that if spillovers are positive, the outside option of all $S \subset N$ is higher in $g$ than in $g^{\prime}$, and if spillovers are negative, these outside options are higher in $g^{\prime}$ than in $g$. These facts are formally established by the following Lemma.

Lemma 1 Let $v$ be anonymous and cohesive. Let $g$ and $g^{\prime}$ be two organizations with set of members $N$, such that $g^{\prime} \subset g$.

i) if $v \in V^{+}$, then $v\left(B,\left\{\pi_{S}, \pi\left(\left.g^{\prime}\right|_{N \backslash S}\right)\right\}\right) \leq v\left(B,\left\{\pi_{S}, \pi\left(\left.g\right|_{N \backslash S}\right)\right\}\right)$ for all $S \subset N, \pi_{S} \in$ $\Pi(S)$ and $B \in \pi_{S}$;

ii) if $v \in V^{-}$, then $v\left(B,\left\{\pi_{S}, \pi\left(\left.g^{\prime}\right|_{N \backslash S}\right)\right\}\right) \geq v\left(B,\left\{\pi_{S}, \pi\left(\left.g\right|_{N \backslash S}\right)\right\}\right)$ for all $S \subset N$, $\pi_{S} \in \Pi(S)$ and $B \in \pi_{S}$.

Proof. Let $S \subset N$. Since $g^{\prime} \subset g$ then $\left.\left.g^{\prime}\right|_{N \backslash S} \subseteq g\right|_{N \backslash S}$. Therefore, if $B^{\prime} \in \pi\left(\left.g^{\prime}\right|_{N \backslash S}\right)$ then either $B^{\prime} \in \pi\left(\left.g\right|_{N \backslash S}\right)$ or $B^{\prime} \subset B$ for some $B \in \pi\left(\left.g\right|_{N \backslash S}\right)$. This follows from the observation that adding a link to a graph never eliminates any of the existing connecting paths between vertices. For the same reason, if $B \in \pi\left(\left.g\right|_{N \backslash S}\right)$ and $B \notin \pi\left(\left.g^{\prime}\right|_{N \backslash S}\right)$ then there exists a sequence of coalitions $\left\{B_{1}^{\prime}, . ., B_{m}^{\prime}\right\} \subset \pi\left(\left.g^{\prime}\right|_{N \backslash S}\right)$ such that $B=\cup_{p=1}^{m} B_{p}^{\prime}$. We conclude that either $\pi\left(\left.g\right|_{N \backslash S}\right)=\pi\left(\left.g^{\prime}\right|_{N \backslash S}\right)$ or $\pi\left(\left.g\right|_{N \backslash S}\right)$ is a concentration of $\pi\left(\left.g^{\prime}\right|_{N \backslash S}\right)$. A direct application of the definitions of positive and negative spillovers implies points i) and ii) of the lemma.

Consider now the implications of lemma 1 for the case of positive spillovers. Since all coalitions' outside options are higher in $g$ than in $g^{\prime}$, if communication is unrestricted all allocations which are stable in $g$ are also stable in $g^{\prime}$. If communication is restricted, this effect on outside option is reinforced by the effect of a larger set of potential defecting coalitions in $g$ than in $g^{\prime}$. These observations lead to the following proposition. 
Proposition 1 Let $v \in V^{+}$be anonymous and cohesive. Let $g$ and $g^{\prime}$ be two organizations with set of members $N$, such that $g^{\prime} \subset g$. Then $\Phi(g, v) \subseteq \Phi\left(g^{\prime}, v\right)$.

Proof. Consider first problems with unrestricted communication, in which the set of potential defecting coalitions is the same in $g$ as in $g^{\prime}$. If $\mathbf{u} \in \Phi(g, v)$ then for all $S$ and all $\pi_{S}$ we have $\sum_{i \in B} u_{i} \geq v\left(B,\left\{\pi_{S}, \pi\left(\left.g\right|_{N \backslash S}\right)\right\}\right)$ for at least one $B \in \pi_{S}$. By Lemma 1 we know that $v\left(B,\left\{\pi_{S}, \pi\left(\left.g\right|_{N \backslash S}\right)\right\}\right) \geq v\left(B,\left\{\pi_{S}, \pi\left(\left.g^{\prime}\right|_{N \backslash S}\right)\right\}\right)$. It follows that $\mathbf{u} \in \Phi\left(g^{\prime}, v\right)$. Consider now problems with restricted communication. Here, the set of coalitions with the ability to defect from $g^{\prime}$ is a strict subset of the set of coalitions with the ability to defect from $g$. This because if a connecting path exists between two vertices in $g^{\prime}$, then the same path exists in $g$. The above argument based on outside options can therefore be applied to the set of connected coalitions in $g^{\prime}$.

Although it is possible to obtain a strict inclusion for at least one partition function with positive spillovers, the next example shows that this is not true in general.

Example 1 Let $N=\{1,2,3\}, g^{\prime}=(N,\{12,13\})$ and $g=(N,\{12,13,23\})$. Let $v$ be as follows:

$$
\begin{aligned}
v(\{i\},\{\{1\},\{2\},\{3\}\}) & =1 \text { for } i=1,2,3 ; \\
v(\{i\},\{\{i\}, N \backslash\{i\}\}) & =1+\varepsilon \text { for } i=1,2,3 ; \\
v(\{1,2\},\{\{1,2\},\{3\}\}) & =v(\{1,3\},\{\{1,3\},\{2\}\})=v(\{2,3\},\{\{2,3\},\{1\}\})=3 ; \\
v(N,\{N\}) & =\frac{9}{2} .
\end{aligned}
$$

The set $\Phi(g, v)$ consists of the single allocation $\left\{\frac{3}{2}, \frac{3}{2}, \frac{3}{2}\right\}$, which also belongs to $\Phi\left(g^{\prime}, v\right)$. Although player 1 has a higher payoff by defecting from $g$ than from $g^{\prime}$, this is not reflected by any allocation in $\Phi(g, v)$ that gives 1 a lower payoff than to the other players.

Consider then the case of negative spillovers. A similar monotonicity property to that established in proposition 1 applies here only in the case of unrestricted communication, where the sole effect of additional links on outside options are relevant (see the proof of proposition 2 below). When communication is restricted, however, additional links enlarge the set of coalitions with the ability to defect. While the effect on outside options plays in favour of stability, this second effect plays against it, breaking down the monotonic relation between higher connectedness of the organization and its stability properties. 
Proposition 2 Let $v \in V^{-}$be anonymous and cohesive. Let $g$ and $g^{\prime}$ be two organizations with set of members $N$, such that $g^{\prime} \subset g$. Under unrestricted communication $\Phi\left(g^{\prime}, v\right) \subseteq$ $\Phi(g, v)$.

Proof. If $\mathbf{u} \in \Phi\left(g^{\prime}, v\right)$ then for all $S$ and all $\pi_{S}$ we have $\sum_{i \in B} u_{i} \geq v\left(B,\left\{\pi_{S}, \pi\left(\left.g^{\prime}\right|_{N \backslash S}\right)\right\}\right)$ for at least one $B \in \pi_{S}$. By Lemma 1 we know that $v\left(B,\left\{\pi_{S}, \pi\left(\left.g^{\prime}\right|_{N \backslash S}\right)\right\}\right) \geq v\left(B,\left\{\pi_{S}, \pi\left(\left.g\right|_{N \backslash S}\right)\right\}\right)$. It follows that $\mathbf{u} \in \Phi(g, v)$.

The following example illustrates the failure of proposition 2 in problems with restricted communication.

Example 2 Let $N=\{1,2,3,4\}$, and $g^{\prime} \subset g$ be two organizations with set of members $N$, such that $L\left(g^{\prime}\right)=\{12,13,14\}, L(g)=\{12,13,14,23,24,34\}$. Let the partition function $v \in V^{-}$be as follows:

$v(1,\{N\})=1 ; v(1,\{1,2,34\})=1-\varepsilon ; v(12,\{12,34\})=2 ; v(12,\{12,34\}) ; v(N,\{N\})=4+\delta$,

with $\varepsilon$ and $\delta$ positive and less than unity. The following allocation is stable for $g^{\prime}$ :

$$
u_{2}=u_{3}=u_{4}=1-\varepsilon ; u_{1}=1+3 \varepsilon+\delta .
$$

It is easy to check that the same allocation is not stable for $g$, since coalition $\{23\}$ has an incentive to defect and get $v(13,\{13,24\})=2>1-2 \varepsilon$. Although having the same incentives, coalition $\{23\}$ has not the ability to defect in $g^{\prime}$

\subsubsection{Implications for Organizational Design}

In this section we discuss which implications can be drawn from the obtained results for the problem of organizational design. In the present context, we can identify organizational design with the choice of a specific connected network $g$ with vertex set $N$. Given that the value $v(N,\{N\})$ generated by the the grandcoalition is independent of the adopted organizational form, we can focus on the effect of design on the stability of cooperation, in the sense made clear in definition 10.

Let us begin with the case of positive spillovers. It is clear from an iteration of proposition 1 that all the allocations that can be sustained as stable in at least one organizational form can also be sustained as stable in some minimally connected network (or tree). Symmetric 
and opposite implications can be drawn for problems with negative spillovers and unrestricted communication, where the set of stable allocations for the complete graph is the union of all allocations which are stable for at least on organizational form. In both classes of spillovers, the adoption of the outlined organizational forms allows the designer to achieve stability by means of the largest possible set of allocations. In other terms, if adopted, these organizational forms allow to achieve stability by imposing the minimal constraints on the payoff allocation.

Although suggesting an intuitive relation between the degree of connectedness of an organization and its stability properties, the above results fail to predict a specific tree architecture under positive spillovers (the choice will depend on the shape of the partition function) and, under negative spillovers, leave the problem of restricted communication unsolved. The next section addresses both problems in the specific class of "egalitarian design problems".

\subsubsection{Stable egalitarian organizations}

This section studies the problem of organizational design when the only allocation available to the designer is the egalitarian payoff vector $\mathbf{u}^{e}(v)$, equally splitting the value $v(N,\{N\})$ among the players in $N$. Definition 10 is rephrased here as follows:

Definition 11 The connected network $g$ with vertex set $N$ is a stable egalitarian organization under the partition function $v$ if $\mathbf{u}^{e}(v) \in \Phi(g, v)$.

Egalitarian design problems are of interest from a normative standpoint when the partition function is anonymous; moreover, as suggested by a recent work of Jackson (2005), the egalitarian allocation should be expected whenever players make distributional arrangements before the network has formed and when its structure is still flexible. As we said, in this paper the restriction to the egalitarian allocation has the additional appeal of allowing for sharp predictions on the architecture chosen by the designer in problems with restricted communication. We will see how, in such problems, positive spillovers imply the choice of a particular minimally connected organization, the star, while the complete network and the circle are predicted under negative spillovers, with equivalent stability properties.

Before turning to these results in more detail, we first consider, for completeness, the case of unrestricted communication. Here, both results of propositions 1 and 2 apply. However, under positive spillovers the inclusion relation turns out to be never strict, so that organizational design plays no role for stability. 
Proposition 3 Let $v \in V^{+}$be anonymous and cohesive. Let $g$ and $g^{\prime}$ be two organizations with set of members $N$. Then $\mathbf{u}^{e} \in \Phi(g, v)$ if and only if $\mathbf{u}^{e} \in \Phi\left(g^{\prime}, v\right)$.

Proof. If $\mathbf{u}^{e} \in \Phi(g, v)$ then, for all $S \subset N$ and for all $\pi_{S}$ there exists $B \in \pi_{S}$ of size $b$ such that

$$
v\left(B,\left\{\pi_{S}, \pi\left(\left.g\right|_{N \backslash S}\right)\right\}\right) \leq b \frac{v(N,\{N\})}{n} .
$$

Suppose that $\mathbf{u}^{e} \notin \Phi\left(g^{\prime}, v\right)$. In this case there exists $S^{\prime} \subset N$ of size $s^{\prime}$ and $\pi_{S^{\prime}}$ such that for all $B^{\prime} \in \pi_{S^{\prime}}$ :

$$
v_{B}\left(B^{\prime},\left\{\pi_{S^{\prime}}, \pi\left(\left.g^{\prime}\right|_{N \backslash S^{\prime}}\right)\right\}\right)>b^{\prime} \frac{v(N,\{N\})}{n},
$$

where $b^{\prime}$ denotes the size of $B^{\prime}$.

We will prove that this leads to a contradiction. Suppose we can find a coalition $S$ of size $s^{\prime}$ such that $\left.g\right|_{N \backslash S}$ is a connected network. Define the permutation $\sigma$ so that $S=\sigma\left(S^{\prime}\right)$. In this case, positive spillovers and anonymity of $v$ would imply that for all $\pi_{S}$ and all $B \in \pi_{S}$ :

$$
v\left(B,\left\{\pi_{S}, \pi\left(\left.g\right|_{N \backslash S}\right)\right\}\right) \geq v\left(\sigma(B),\left\{\sigma\left(\pi_{S}\right), \pi\left(\left.g^{\prime}\right|_{N \backslash \sigma(S)}\right)\right\}\right) .
$$

Conditions (2) and (3) contradict condition (1) when applied to coalition $S$.

To prove the result we therefore just need to prove that we can find such a coalition $S$. This directly follows from the fact that if $g$ is connected, then for all $k=1,2, \ldots, n$ we can find a set of vertices $S$ of size $k$ which is not a vertex-cut for $g$. This fact can be shown as follows by induction on $k$. For $k=1$, let $i$ be the endpoint of a maximal path in $g$ (there must always exists such a path since $g$ is connected and $N$ is finite). Since it is an endpoint, then all the vertices adjacent to $i$ must belong to that path, which is still connected in $\left.g\right|_{N \backslash\{i\}}$. It follows that the network $\left.g\right|_{N \backslash\{i\}}$ is itself connected. Suppose now that the hypothesis is true for $k=m$. Then there exists a set $S$ of size $m$ such that $\left.g\right|_{N \backslash S}$ is connected. We now apply again the result for $k=1$ to say that there exists some $i \in N \backslash S$ which is not a cut vertex for $\left.g\right|_{N \backslash S}$. Consider then the coalition $S \cup i$. This coalition is of size $m+1$, and, by the fact that $\left.g\right|_{N \backslash S}$ is connected, we conclude that $\left.g\right|_{N \backslash(S \cup\{i\})}$ is connected, which proves the result.

The same indeterminacy results does not hold for the class of negative spillovers, where the inclusion proved in proposition 2 can be strict, as shown in the following example:

Example 3 Under the following partition function (with negative spillovers), the efficient egalitarian allocation is stable in a 4 vertex complete graph, but is not stable in a 4 vertex 
incomplete graph.

$$
\begin{aligned}
v(\{i\} ;\{i, j k l\}) & =1 ; v(\{i\} ;\{\{i\},\{j, k\},\{l\}\})=2 ; \\
v(\{i, j\} ;\{\{i, j\},\{k, l\}\}) & =4 ; v(\{k, l\} ;\{\{i\},\{j\},\{k, l\}\})=7 ; \\
v(\{i, j, k\} ;\{\{i, j, k\},\{l\}\}) & =8 ; v(\{i, j, k, l\},\{i, j, k, l\})=12 .
\end{aligned}
$$

We have that $u^{e}(v)=(3,3,3)$. Consider first the complete graph. Each single player, by defecting from the complete graph gets 1 , each coalition of size 2 gets 4 , and each coalition of size 3 gets 8 . Therefore, the complete graph is a stable egalitarian organization. Consider now the graph in which only the link $i j$ is missing. A defection by coalition $(k l)$ induces the partition $\{\{i\},\{j\},\{k, l\}\}$ in which coalition $\{k, l\}$ obtains a payoff of $7>6=2 u^{e}(v)$. Therefore, this incomplete egalitarian organization is not stable.

We now turn to the more complex and interesting case of restricted communication. Proposition 4 studies the case of positive spillovers, where additional links strengthen both the incentives and the ability to defect. The restriction to the egalitarian allocation allows a refinement of the result of proposition 1 , selecting a unique minimally connected organization - the star.

Proposition 4 Let $v \in V^{+}$be anonymous and cohesive. Let $g^{*}$ be a star organization with set of vertices $N$. Under restricted communication the following facts hold:

i) $\mathbf{u}^{e}(v) \in \Phi(g, v)$ implies $\mathbf{u}^{e}(v) \in \Phi\left(g^{*}, v\right)$, for all organizations $g \neq g^{*}$ such that $N(g)=$ $N$.

ii) If $n \geq 4$ there exists a function $v \in V^{+}$such that $\mathbf{u}^{e}(v) \in \Phi\left(g^{*}, v\right)$ but $\mathbf{u}^{e}(v) \notin \Phi(g, v)$ for all organizations $g \neq g^{*}$ such that $N(g)=N$.

Proof. i) Consider first defections of single vertex. Note first that since $g$ is connected, it must admit at least two vertices which are not cut-vertices. ${ }^{8}$ Let $h$ denote one such vertex. Note then that $u^{e}(v) \in \Phi(g, v)$ implies:

$$
u^{e}(v) \geq v(\{h\},\{\{h\}, N \backslash\{h\}\}) .
$$

\footnotetext{
${ }^{8}$ This standard graph theoretic fact can be easily proved as follows. Let $i$ be the endpoint of a maximal path in $g$ (there must always exists such a path since $g$ is connected). Since it is an endpoint, then all the vertices adjacent to $i$ must belong to that path, which is still connected in $\left.g\right|_{N \backslash\{i\}}$. It follows that the if $g$ is connected then the graph $\left.g\right|_{N \backslash\{i\}}$ is connected too.
} 
Positive spillovers and anonymity imply that for all $\pi \in \Pi(N)$ and all $i \in N$ :

$$
v(\{h\},\{\{h\}, N \backslash\{h\}\}) \geq v(\{i\}, \pi) .
$$

We conclude that no player has an incentive to defect from $g^{*}$.

Consider now defections of coalitions of more than one player. Let $S$ be a coalition of size $s \geq 2$ connected in $g^{*}$. This coalition must include the central player in the star, since no link among peripheral players exists in $g^{*}$. As a consequence, the partition $\pi\left(\left.g^{*}\right|_{N \backslash S}\right)$ induced on $N \backslash S$ by the defection of $S$ from $g^{*}$ is made of all singletons in $N \backslash S$, and all other partitions of the set $N \backslash S$ are a concentration of $\pi\left(\left.g^{*}\right|_{N \backslash S}\right)$. Let now $S^{\prime}$ be a coalition of size $s$ and define a permutation $\sigma$ such that $S^{\prime}=\sigma(S)$. By anonymity of $v$, for all $\pi_{S}$ and all $B \in \pi_{S}$, positive spillovers imply that

$$
v\left(B,\left\{\pi_{S}, \pi\left(\left.g^{*}\right|_{N \backslash S}\right)\right\}\right) \leq v\left(\sigma(B),\left\{\sigma\left(\pi_{S}\right), \pi\left(\left.g\right|_{N \backslash \sigma(S)}\right)\right\}\right) .
$$

But since $g$ is stable by assumption, we have that at least one coalition $\sigma(B) \in \sigma\left(\pi_{S}\right)$ satisfied the following condition:

$$
v\left(\sigma(B),\left\{\sigma\left(\pi_{S}\right), \pi\left(\left.g\right|_{N \backslash \sigma(S)}\right)\right\}\right) \leq s \cdot u^{e}(v) .
$$

Inequalities (6) and (7) directly imply that coalition $S$ has no incentives to defect from $g^{*}$.

Therefore, in order to show that $g^{*}$ is stable we just need to show that for each coalition $S$ connected in $g^{*}$ we can find a coalition $S^{\prime}$ of the same size as $S$ which is connected in $g$. Since one such coalition exists in $g^{*}$ for all sizes $s=1,2, \ldots, n$, this adds up to showing that one connected coalition $S_{k}$ of size $k$ exists as well in $g$ for all $k=1,2, \ldots, n$. This standard graph theoretic fact can be proved as follows. Since $g$ is connected, there must be a vertex $i$ in $g$ such that $\left.g\right|_{N \backslash\{i\}}$ is a connected graph of size $(n-1)$ (see the proof in footnote ??). By the same argument, there must be some vertex $j$ in $\left.g\right|_{N \backslash\{i\}}$, so that $\left.g\right|_{N \backslash\{i \cup j\}}$ is a connected graph of size $(n-2)$. And so on until size 1 . So, connectedness of $g$ implies that for each size $k=1,2, \ldots, n$, there exists a coalition $S_{k}$ of size $k$ that is connected in $g$.

ii) We proceed by first showing that for all $g \neq g^{*}$ a coalition $T$ of size $t=2$ can be found for which the induced partition $\pi\left(\left.g\right|_{N \backslash T}\right)$ is not made of all singletons. The proof goes as follows: consider a coalition $T=\{i, j\}$. Either $\pi\left(\left.g\right|_{N \backslash T}\right)$ contains a coalition $S$ with $s \geq 2$, in which case the desired result obtains, or $\pi\left(\left.g\right|_{N \backslash T}\right)$ contains only singletons, meaning that the subgraph $\left.g\right|_{N \backslash T}$ is empty. Since $g$ is connected, all vertices in $N \backslash T$ must be linked in $g$ 
with either $i$ or $j$ or both. If all vertices in $N \backslash T$ are linked with the same vertex in $\{i, j\}$, then $g$ is a star. We therefore consider the case in which at least one vertex $h(i)$ is linked with $i$ and at least one vertex $h(j)$ is linked with $j$. In this case, $\{i, h(i)\}$ serves as the desired coalition $T$, since the graph $\left.g\right|_{N \backslash\{i, h(i)\}}$ contains the link $j h(j)$, meaning that the partition $\pi\left(\left.g\right|_{N \backslash\{i, h(i)\}}\right)$ is not made of all singletons.

Consider now a partition function $v \in V^{+}$satisfying the following constraints:

$$
\begin{aligned}
v(\{i\},\{\{i\}, N \backslash\{i\}\}) & =\frac{v(N,\{N\})}{n} ; \\
v\left(T,\left\{T, \bar{\pi}_{N \backslash T}\right\}\right) & =2 \frac{v(N,\{N\})}{n} \forall T: t=2 ; \\
v\left(S,\left\{S, \bar{\pi}_{N \backslash S}\right\}\right) & \leq s \frac{v(N,\{N\})}{n} \forall S: s>2,
\end{aligned}
$$

where for all $S \subset N$ the notation $\bar{\pi}_{N \backslash S}$ is used to denote the singletons partition. It is clear that $u^{e}(v) \in \Phi\left(g^{*}, v\right)$. Also, since we have shown that for all $g \neq g^{*}$ there exists a coalition $T_{g}$ of size $t=2$ for which $\pi\left(\left.g\right|_{N \backslash T_{g}}\right)$ is not made of all singletons, positive spillovers imply:

$$
v\left(T_{g},\left\{T_{g}, \pi\left(\left.g\right|_{N \backslash T_{g}}\right)\right\}\right)>2 \frac{v(N,\{N\})}{n},
$$

which implies in turns that $u^{e}(v) \notin \Phi(g, v)$.

Proposition 5 studies the case of negative spillovers. Here, the opposite effects of additional links on the incentives and the ability to defect generate a trade off with two equilibrium points: an incomplete structure, the circle, and the complete graph.

Proposition 5 Let $v \in V^{-}$be anonymous and cohesive. Let $g^{c}$ be a circle organization with set of members $N$. Under restricted communication the following facts hold:

i) $u^{e}(v) \in \Phi(g, v)$ implies $u^{e}(v) \in \Phi\left(g^{c}, v\right)$, for all non complete organizations $g \neq g^{c}$ such that $N(g)=N$.

ii) There exists a value function $v \in V^{-}$such that $u^{e}(v) \in \Phi\left(g^{c}, v\right)$ and $u^{e}(v) \notin \Phi(g, v)$, for all non complete organizations $g \neq g^{c}$ such that $N(g)=N$.

iii) $u^{e}(v) \in \Phi\left(g^{c}, v\right)$ if and only if $u^{e}(v) \in \Phi\left(g^{N}, v\right)$.

Proof. i) Suppose $u^{e}(v) \in \Phi(g, v)$. Let $S$ be a coalition of size $s$ connected in $g$. Then, for all $\pi_{S} \in \Pi(S)$ there exists $B \in \pi_{S}$ of size $b$ such that

$$
v\left(B,\left\{\pi_{S}, \pi\left(\left.g\right|_{N \backslash S}\right)\right\}\right) \leq b \cdot u^{e}(v) .
$$


Such connected coalition $S$ exists in $g$ for all $s=1,2, \ldots, n$.

Consider now a connected coalition $S^{\prime}$ in $g^{c}$ of size $s$. By the structural properties of $g^{c}$ we have that $\left.g^{c}\right|_{N \backslash S^{\prime}}$ is connected, so that $\pi\left(\left.g^{c}\right|_{N \backslash S^{\prime}}\right)=\left\{N \backslash S^{\prime}\right\}$. Define now the permutation $\sigma$ such that $S^{\prime}=\sigma(S)$, where $S$ is a connected coalition in $g$. Anonymity and negative spillovers of $v$, together with and the fact that $\left\{N \backslash S^{\prime}\right\}$ is a concentration of all partitions of the set $N \backslash S^{\prime}$, imply that for all $\pi_{S}$ and all $B \in \pi_{S}$ we have:

$$
v\left(B,\left\{\pi_{S}, \pi\left(\left.g\right|_{N \backslash S}\right)\right\}\right) \geq v\left(\sigma(S),\left\{\sigma\left(\pi_{S}\right), \pi\left(\left.g^{c}\right|_{N \backslash \sigma(S)}\right)\right\}\right) .
$$

It follows from propositions 8 and 9 that $u^{e}(v) \in \Phi\left(g^{c}, v\right)$.

ii) We next show that there exists a partition function $v$ such that $\mathbf{u}^{e}(v) \in \Phi\left(g^{c}, v\right)$ and $\mathbf{u}^{e}(v) \notin \Phi(g, v)$ for all incomplete connected networks $g \neq g^{c}$.

We proceed by first showing that for all such $g$ there exists a coalition $S$ which is connected in $g$ and such that the induced subgraph $\left.g\right|_{N \backslash S}$ is disconnected. Since $g$ is not complete, there exist three vertices, say $i, j, k$, inducing a line graph, that is, such that $\left.g\right|_{\{i, j, k\}}=$ $(\{i, j, k\},\{i j, j k\})$. Suppose first that $j$ has degree greater than 2, i.e., that there are more than two vertices linked with $j$ in $g$. Defined the set $H$ as the set of all vertices $h$ other than $i$ and $k$ for which there exists at least one path joining $h$ and $j$ that does not include $i$ nor $k$ :

$$
H=\{h \in N \backslash\{i, k\}: \exists P(h, j) \subset N \backslash\{i, k\}\} .
$$

Let $\bar{H} \equiv N \backslash H$. If the graph $\left.g\right|_{N \backslash(\bar{H} \cup\{j\})}$ is disconnected, then the set $H \cup\{j\}$ is the required connected set in $g$. If the graph $\left.g\right|_{N \backslash(\bar{H} \cup\{j\})}$ is connected, then $\{i, j, k\}$ is the required connected set in $g$.

To complete the argument, we need to consider the case in which every inner vertex of every induced line graph of three vertices in $g$ has degree 2 . Consider again the line $i, j, k$, and suppose, by contradiction, that $j$ is not a cut vertex of $g$. In this case, there exists a path in $g$ connecting $i$ and $k$ that does not include $j$. Among all such paths, consider the shortest one. It is clear that each vertex in this path is the inner vertex of a three vertex line, and must therefore be of degree 2. This, together with the fact that $g$ is connected, implies that $g$ must be a wheel.

Consider now the partition function $v \in V^{-}$constructed as follows:

$$
v(S,\{S, N \backslash S\})=s \cdot u^{e}(v)>\sum_{B \in \pi_{S}} v\left(B,\left\{\pi_{S}, N \backslash S\right\}\right) \forall S \subset N, \forall \pi_{S} \in \Pi(S) .
$$


It is clear that $u^{e}(v) \in \Phi\left(g^{c}, v\right)$. Let now $T$ be the coalition of size $t$ which is connected in $g$ and such that $\left.g\right|_{N \backslash T}$ is disconnected. Define by $\sigma$ the permutation such that $T=\sigma\left(T^{\prime}\right)$ for some $T^{\prime}$ connected in $g^{c}$ (we know that such $T^{\prime}$ can be found since there exists a connected coalition in $g^{c}$ for all sizes). By negative spillovers, and by the fact that $\left\{N \backslash T^{\prime}\right\}$ is a concentration of $\pi\left(\left.g\right|_{N \backslash T}\right)$, we obtain:

$$
v\left(T^{\prime},\left\{\pi_{T^{\prime}}, \pi\left(\left.g^{c}\right|_{N \backslash T^{\prime}}\right)\right\}\right)<v\left(T,\left\{\pi_{T}, \pi\left(\left.g\right|_{N \backslash T}\right)\right\}\right)
$$

from which we conclude that $u^{e}(v) \notin \Phi(g, v)$.

iii) Note that all defections by a connected coalition $S$ in $g^{N}$ induces the same partition on $N \backslash S$ as $S$ would induce by defecting from $g^{c}$ is $S$ were connected in $g^{c}$. Since there exists a connected coalition in $g^{c}$ for all possible sizes $s=1,2, \ldots, n$, the result follows.

The obtained results can be again read in terms of organizational design. If spillovers are positive, the designer will achieve stability "more often" by adopting a star organizational form, since the set of partition functions that admit stability under the star architecture strictly includes the set of all partition functions that admit stability under any other architecture. If spillovers are negative, the same property is enjoyed by the circle and the complete architectures which are, in terms of stability, equivalent.

\subsection{Concluding Remarks}

We have studied the relation between the organizational form adopted by a group of cooperating agents and the stability of cooperation. The organizational form (formally represented by a connected network) affects both the incentives to defect - by determining the extent of cooperation after the defection - and the ability to defect - by determining the amount of communication available to organizational members. The analysis can be applied to the design of all those socioeconomic organizations, such as firms, international organizations, economic unions, political parties, from which members can at any time step out and interact in a non cooperative manner.

We have found that certain basic structural features of the network can be related to the stability of cooperation. Under positive spillovers, sparse organizations are more stable than dense ones, while the opposite holds under negative spillovers. For the specific case in which cooperation gains are split equally, the star and the circle turn out to have maximal stability properties under positive and negative spillovers, respectively. 
We finally wish to stress two important simplifications underlying the present analysis. First, the adoption of a partition function has set aside all efficiency issues in designing the organizational form. In the presence of such issues, the architecture identified in this paper may fail to be efficient, and, according to our notion of stability, stable. Nevertheless, the qualitative result that relates the cohesion of the cooperating group with its stability would remain valid, although with less extreme implications. Second, players are assumed not to be able to modify the network if not by disconnecting from it. For this reason, this paper is not a contribution to the literature on network formation, where the incentives to form and sever links are determined by an allocation rule which is sensible to the shape of the network (see Jackson and van den Nouweland (2005) for an analysis of network stability with respect to coalitional deviations).

A closer relation can be drawn with the literature on games of coalition formation of the type first introduced by Hart and Kurz (1983) and then applied to the context of games in partition function (see the surveys of Yi (2003) and Bloch (2003)). The relation between a graph and the induced coalition structure used in this paper can be viewed as a variation of the "gamma" and "delta" coalition formation rules considered in Hart and Kurz. While in that paper coalitions are associated only with completely connected subgraphs, in the present paper coalitions can have an incomplete (but connected) architecture, and the relation between this architecture and the stability of the coalitions becomes relevant. One corollary of proposition 3 is that the conditions under which an egalitarian organization is stable are the same under which the grand coalition is induced as a strong Nash equilibrium of the "delta" game of Hart and Kurz (1983) played under the coalition-wise egalitarian payoff allocation rule.

\section{References}

[1] Arrow K. J. (1974), The Limits of Organization, W.W. Norton \& Company Ed.

[2] Bolton, P., Dewatripont, M. (1994) The Firm as a Communication Network. Quarterly Journal of Economics 109: 809-839.

[3] Bloch, F. (2003) Non Cooperative Models of Coalition Formation in Games with Spillovers, in C. Carraro (ed.) The endogenous formation of economic coalitions, Fon- 
dazione Eni Enrico Mattei Series on Economics and the Environment, Cheltenham, U.K. and Northampton, Mass.: Elgar.

[4] Chander, A. (1966) Strategy and Structure, New York, Doubleday.

[5] Demange, G. (2004) On Group Stability in Hierarchies and Networks. Journal of Political Economy 112(4): 754-778.

[6] Hart, S. and M. Kurz (1983) Endogenous Formation of Coalitions. Econometrica 51(4): 1047-1064

[7] Holmstrom R and P . Milgrom, (1991) Multi-Task Principal-Agent Problems: Incentive Contracts, Asset Ownership and Job Design. Journal of Law, Economics and Organization, Vol 7, Special Issue.

[8] Holmstrom, R. and Milgrom, P. (1994) The Firm as an Incentive System American Economic Review, 84(4): 972-91.

[9] Ichiishi, T., (1981) A Social Coalitional Equilibrium Lemma. Econometrica 49(2): 369377

[10] Jackson, M.O. (2005) Allocation Rules for Network Games. Games and Economic Behaviour 51(1): 128-154.

[11] Jackson, M.O. and van den Nouweland (2005). Strongly Stable Networks. Games and Economic Behaviour 51(2): 420-444.

[12] Myerson, R. (1977) Graphs and Cooperation in Games. Math.Oper. Res. 2: 225-229.

[13] Radner, R. (1993). The Organization of Decentralized Information Processing. Econometrica 61: 1109-46.

[14] Ray, D., Vohra, R. (1997) Equilibrium Binding Agreements. Journal of Economic Theory 73: $30-78$.

[15] Yi, S.- S. (1997) Stable Coalition Structure with Externalities. Games and Economic Behaviour 20: 201-237. 
[16] Yi, S. S., (2003) The Endogenous Formation of Economic Coalitions: The Partition Function Approach, in C. Carraro (ed.) The endogenous formation of economic coalitions, Fondazione Eni Enrico Mattei Series on Economics and the Environment, Cheltenham, U.K. and Northampton, Mass.: Elgar. 